\title{
Spinal Motion Segments - II: Tuning and Optimisation for Biofidelic Performance
}

\author{
Constantinos Franceskides ${ }^{1}$, Emily Arnold ${ }^{1}$, Ian Horsfall ${ }^{2}$, Gianluca Tozzi ${ }^{3}$, Michael C. \\ Gibson $^{4}$, Peter Zioupos ${ }^{1^{*}}$ \\ 1. Musculoskeletal and Medicolegal Research Group, Cranfield Forensic Institute, Cranfield University, Defence \\ Academy of the UK; Shrivenham, SN6 8LA, UK \\ 2. Centre for Defence Engineering, Cranfield University, Defence Academy of the UK; Shrivenham, SN6 8LA, UK \\ 3. School of Engineering, University of Portsmouth, Portsmouth, PO1 3DJ, UK \\ 4. Centre for Simulation \& Analytics, Cranfield University, Defence Academy of the UK; Shrivenham, SN6 8LA, UK
}

*Corresponding author: P.Zioupos

E-mail: p.zioupos@hotmail.co.uk 


\begin{abstract}
Most commercially available spine analogues are not intended for biomechanical testing, and the few that are suitable for using in conjunction with implants and devices to allow a hands on practice on operative procedures are very expensive and still none of these offers patient-specific analogues that are can be accessed within reasonable time and price range. Man-made spine analogues would also avoid the ethical restrictions surrounding the use of biological specimens and complications arising from their inherent biological variability. Here we sought to improve the biofidelity and accuracy of a patient-specific motion segment analogue that we presented recently [13]. These models were made by acrylonitrile butadiene styrene (ABS) in 3D printing of porcine spine segments (T12-L5) from microCT scan data, and were tested in axial loading at $0.6 \mathrm{~mm} / \mathrm{min}$ (strain rate range $6-10 \times 10^{-4} \mathrm{~s}^{-1}$ ). In this paper we have sought to improve the biofidelity of these analogue models by concentrating in improving the two most critical aspects of the mechanical behaviour: the material used for the intervertebral disc and the influence of the facet joints. The deformations were followed by use of DIC (Digital Image Correlation) and consequently different scanning resolutions and data acquisition techniques were also explored and compared to determine their effect. We found that the selection of an appropriate intervertebral disc simulant (PT Flex 85) achieved a realistic force/displacement response and also that the facet joints have a key role to play to achieve a biofidelic behaviour for the entire motion segment. We have therefore overall confirmed the feasibility of producing, by rapid and inexpensive 3D-printing methods, high-quality patient-specific spine analogue models suitable for biomechanical testing and practice.
\end{abstract}

Keywords: spine, bone analogue, micro-CT, 3D printing, digital image correlation (DIC) 


\section{Introduction}

The demands for hands on training, experimental testing and validation of implants, in conjunction with the bones on which they are applied to, has increased the demand for more accurate analogues that are suitable for biomechanical testing [1]. Analogues circumvent the challenges associated with tests on cadaveric materials, including availability, storing, variation in ambient conditions, handling/disposal issues, as well as ethical restrictions [2-5].

Mechanically and anatomically some models are provided by companies such as Sawbones (USA) and Synbone (Switzerland), these are informative and allow hands on practice, but they are expensive and generic, meaning they are not patient specific [6]. Such analogues provide an alternative to human or animal cadavers for implant testing [7]. They also emulate human cadaveric spines in both morphological and biomechanical terms, such as the range of motion (focusing on bending, twisting, flexing and extending) without, however, addressing exactly the actual compressive behaviour [8-11].

We have previously reported the development of biofidelic models of an analogue porcine motion segment [12,13] using the versatility offered by modern D printing technology [14-16]. These models had vertebrae which matched their biological counterparts pretty accurately for load/displacement behaviour. However, as data acquisition by digital image correlation (DIC) showed there were two aspects of the analogues which needed further refinement and fine tuning: namely the IVD material (intervertebral discs) and the role the facet joints potentially play in the overall behaviour of the motion segment $[17,18]$. It is important to determine the best practices for the incorporation of such elements into analogue models in order to emulate cadaveric samples more accurately. It is also necessary to consider the resolution of scanning and how this affects the analogue's performance.

Here we developed a novel and accessible protocol based on our earlier methods $[12,13]$ involving the inexpensive production of 3D-printed analogues augmented with realistic facet joints and IVDs. Such analogues can be applied in future research projects to develop more complex and accurate models of the musculoskeletal system. 


\section{Materials and methods}

\subsection{Analogue creation}

The templates for the motion segment analogues were porcine spinal material (from animals 812 months old, destined for the food supply chain) obtained from a local butcher. The cadaveric material was de-fleshed with scalpels at $40^{\circ} \mathrm{C}$ in a water bath for $90 \mathrm{~min}[12,13]$. The cleaned samples were scanned using a Nikon CT H225 cone beam $\mu$ CT scanner (X-Tek Systems Ltd, $\mathrm{UK})$ at $70 \mathrm{kV}$ and $90 \mu \mathrm{A}$.

After scanning the cadaveric spinal portions (defined here as real motion segments and represented by the abbreviation RMS), noise reduction and beam hardening corrections were applied to the data using CT Pro 3D (Nikon Metrology UK Ltd, UK). Further reconstruction and editing were carried out using Simpleware ScanIP M-2017.06-SP2 (Synopsys Inc., USA). This process also yielded over-threshold and under-threshold samples which were derived by altering the volume fraction (VF) of the normal analogue by $\pm 40 \%$. The data were then used to create artificial motion segments (represented by the abbreviation AMS) containing artificial vertebral bodies (AVBs).

Four real motion segments were tested, named RMS4-7 (Table 1). The effect of varying the scanning resolution for RMS5-7 was tested by producing two distinct voxel dimensions of 0.063 and $0.123 \mathrm{~mm}$ (RMS\#_N for the normal scans and RMS\#_L for the low magnification /larger voxel size), thus replicating the artificial vertebral bodies (AVB5-7). In contrast, RMS4 was used as a model to select disc simulants (AMS8_70 and AMS8_85). In addition, facet stiffness was tested simply by removing the facet processes from the tested models (AMS\#_F denoting the presence of a facet and AMS\#_NF denoting facet absence). Finally, the overcompensation and undercompensation of thresholding was tested using RMS4 samples (AVB\#_O for overcompensation and AVB\#_U for undercompensation). This was carried out using the Grey Scale (GS) selection tools in ScanIP and adjusting as required to increase or decrease the VF (volume fraction appropriately) variance. Finally, operations such as flood-fill as well as a smoothing filter (Recursive Gaussian at 0.2 pixels) were applied to finish off the post-printed models. 
The CT (DICOM) data were imported as 32-bit float, converted to 8-bit float and then resampled to $0.400 \mathrm{~mm}$ in an effort to reduce the file size, to match the printer nozzle size, and also to be in line with our earlier work on this topic [7]. The analogues were then printed using a fused deposition modelling (FDM) UPrintSE 3D printer (Stratasys Inc., USA). The .cmb toolpath files were created using Stratasys CatalystEX v4.5 (Stratasys, Inc.) with the support fill set to 'smart' and the model interior to 'solid', meaning that the average printing time per sample was $\sim 40$ min for the larger AVB samples.

Finally, the IVD was formed using PT Flex liquid rubber formulations (Polytek Development Corp., USA): PT Flex 85 of Shore A hardness=85 [19], and the intra-facet cartilage was formed using PT Flex 70 of Shore A hardness=70. Once mixed, the rubber was injected into a cast created around the superior and inferior endplates and the superior and inferior articular processes of the IVDs using Sugru mouldable glue (FormFormForm Ltd, London, UK). The IVD thickness was based on the CT reconstruction data and was achieved by placing struts of the appropriate height (produced using the matching PT Flex compound) at three points separating the endplates.

\subsection{Sensitivity and refinement of data acquisition techniques}

\subsubsection{Strain validation}

The DIC method was tested against a 25 x $9 \mathrm{~mm}$ polyvinyl chloride (PVC) core with a speckled pattern applied using high-contrast paint (Fig. 1). A sequence of images was captured at $1000 \mathrm{fps}$ using Phantom V1212 and V2512 high-speed cameras (Vision Research Inc., USA). PVC was used instead of ABS due to its availability, given that the Young's modulus and tensile strength of both materials are similar (Matmatch, Germany). The DIC data were analysed using GOM Correlate Professional 2017 Hotfix 5 (GOM GmbH, Germany).

\subsubsection{Facet size and point distance sensitivity study}

To measure the effect of the facet size and point distance, the results from a 'strain gauge' (a 6mm gauge length contact extensometer, EPSILON Tech, Jackson, WY 83001, USA) were considered as the benchmark and thus compared to the results obtained from GOM. To do this, 
the results from the default point distance (16 pixels) and facet size (19x19 pixels) were used to compare the default point distance to facet size ratio (16/19) to other ratios $(4 / 3,3 / 3,1 / 2$ and 1/3). The results were then compared to the strain gauge results and their variances were plotted. In a similar manner, the different facet sizes $(6,12,19,24$ and 30) were tested with varying point distances and the results were then compared to the strain gauge data to obtain the variances. The different size to point distance ratios (lowest common multiplier, LCM) tested against the facet sizes are shown in Table 2.

\subsection{Dead weight experiments}

The motion segments were incrementally loaded to determine whether residual effects under constant loading were identified by DIC analysis. This was achieved by looking at the displacement in the $x$ and $y$ axes along the entire length of the sample. Loads were applied in increments of $100 \mathrm{~N}$ up to $2000 \mathrm{~N}$, with a series of 10 images captured once the sample held the constant load for $1 \mathrm{~h}$. The testing method and apparatus are shown in Fig. 2. The sample was loaded directly under the push rod and then weight was added on top to achieve the necessary load increments.

\subsection{Effects of support cleaning apparatus}

A support cleaning apparatus (PADT Inc., USA) combined with WaterWorks P400SC (Stratasys Inc.) is the preferred method for cleaning/removing the support material from 3D-printed components, and this is essential for intricate and complex geometries, particularly where mechanical responses are important. The supports were removed by immersion in $\mathrm{NaOH}$ at $70 \mathrm{C}^{\circ}$ for $4 \mathrm{~h}$ as recommended by the manufacturer. Fig. 3 shows a mask created (shown in green) to highlight the support within the vertebral body. The figure also serves to illustrate the recreation of the inner trabecular structure of a vertebral body which is made possible with 3D printing. To test the importance of cleaning, two analogues were printed. One was subjected to the cleaning treatment and the other was used as an untreated control for subsequent testing to failure. 


\subsection{Testing of analogue specimens}

All compressive testing was conducted using an Instron 5567 tensile testing machine (Instron, UK) fitted with a $10-\mathrm{kN}$ load cell, and each sample was compressed at a quasi-static loading rate of $0.6 \mathrm{~mm} / \mathrm{min}$ (strain rate range $6-10 \times 10-4 \mathrm{~s}^{-1}$ ). A relatively small preload of $10-50 \mathrm{~N}$ was applied to all samples before initiating the test to reduce any inherent contact errors [14]. A 75$\mathrm{mm}$ spherical joint platen was used to minimise contact errors and bending moments, but also to ensure consistent loading across the sample surface area. DIC analysis was conducted by capturing images at $1000 \mathrm{fps}$ using the Phantom high-speed cameras described above controlled by PCC v2.8.761 software (Vision Research Inc.).

\section{Results and discussion}

\subsection{DIC sensitivity, strain validation and dead weight error}

The tests conducted on varying facet sizes, point distances and their associated ratios are shown in Fig. 4 (a) and (b). The highest ratio of 4/3 (or as per LCM, 608/456) was unable to yield strains in two cases, when the facet sizes were 24 and 30, probably because the intersection deviation was outside computational limits. In addition, the worst ratio when comparing average errors was $1 / 3$ (or as per LCM, 152/456), partly due to the error of $4.32 \%$ obtained on the sixpixel facet. All average errors were closely grouped: 1.39\%, 1.70\%, 1.34\%, 1.61\% and 1.73\%, respectively. Fig. $4 \mathrm{~b}$ highlights the error observed when keeping the facet size fixed and varying the point distance (thus altering the facet to point distance ratio). Clearly, the six-pixel facet size resulted in the lowest resolution, probably because the speckle pattern becomes less resolvable with smaller facet sizes, resulting in total colour patching (only white or black within a visible single facet). Hence the recommended speckle size should not be greater than 3-5 pixels [15].

Average errors varied from $2.84 \%$ down to $1.15 \%$ when compared to the strain gauge readings. Facet size provided a consistent low error with the best reading obtained at the 16/19 ratio (or as per LCM, 384/456), which is the ratio recommended by GOM. Results obtained with the ratios 4/3 (as per LCM, 608/456) and 3/3 (as per LCM, 456/456) were less reliable because they provided no data overlap and therefore no intersection, making the facet-to-facet interpolation at 
every stage even less accurate. Even so, the ratio selected for our remaining experiments was the one recommended in the literature, as was the facet size. This achieved the lowest average error and also the lowest individual error of $0.77 \%$.

Fig. 5 shows the plot obtained from the PVC compression tests, revealing clear agreement between the DIC and strain gauge data, with the average error being $9.2 \mu \varepsilon$ and shown by the dashed green line in Fig. 5. Although the error was based on a 0.3-mm compression, the agreement between the two techniques was within anticipated limits [16].

Finally, the noise in the system was recorded as previously described [10]. This was determined in the static state without loading (Fig. 6) and in the static state loaded with "dead weight" (Fig. 7). The in-plane strains (pseudo-strains) were therefore ignored because no external factor was acting against the component so no in-line movements were anticipated. The error obtained from a $20 \times 20 \mathrm{~mm}$ aperture on the $x$ and $y$ axes is shown in Fig. 6. Along the $x$ axis, the error ranges from -7.1 to $1.35 \mu \mathrm{m}$ and along the $y$ axis it ranges from -7.4 to $1.2 \mu \mathrm{m}$, which is less than $2 \mu \mathrm{m}$ over the 20-mm test length and can therefore be disregarded. The dead weight error was calculated only for the direction of loading $(y)$ but also with a $20-\mathrm{mm}$ aperture. The error was acquired by averaging the displacement observed along this axis over the sequence of a 10-image burst at $1000 \mathrm{fps}$. The loading was introduced in increments of $100 \mathrm{~N}$ at 60 -min intervals up to and including $2000 \mathrm{~N}$. As shown in Fig. 7, the displacement varied from -2.1 to $2.7 \mu \mathrm{m}$, which is twice the displacement under no load. This was not expected, yet there seems to be a periodic tendency which may indicate that external factors contribute to the effect. One uncontrolled variable was the heating of the test area, which has an unknown periodicity. This should not be ignored because the radiant heat from the elements could have affected the disc temperature and thus the loading response of the polymer. Regardless of the factors that may have contributed to the variance we observed, the resulting peak-to-peak error was within the range observed in previous studies, i.e. less than $20 \mu \mathrm{m}$ [17]. These results provide confidence in the DIC method, which could be fine-tuned to increase its accuracy. The most important factors include but are not limited to the speckle pattern, the facet size and the point distance, which ensure a fine balance between computational speed and accurate image analysis. 


\subsection{Effects of the support removal process}

Due to the intricate geometry of vertebral bodies and their internal architecture, the toolpath creation software (Stratasys CatalystEX) infilled areas using a soluble support as the analogues were printed. The supports were removed by immersion in $\mathrm{NaOH}$ at $70 \mathrm{C}^{\circ}$ for $4 \mathrm{~h}$. We then conducted a test to compare treated and untreated vertebral bodies in order to determine any effects the support cleaning apparatus might have on their mechanical behaviour under quasistatic compression. Fig. 8 shows the responses of the samples. The recorded stiffness values were 12,838 and $12,763 \mathrm{~N} / \mathrm{mm}$ for the treated and untreated samples, respectively. It is unclear whether this small variance is purely due to the response of the support, or whether it includes the effect of the bath on the material or a combination of both factors. It is not currently possible to test the different options directly because the printing software does not allow printing without the support, thus cleaning is an essential process for support removal.

\subsection{Threshold and low-resolution scanning effects}

Resampling was required for the AVBs to mimic the responses of their biological counterparts as previously reported [7]. The VF was therefore increased, and tests were conducted to determine whether this affected the stiffness of the AVBs and thus their response under loading. Table 3 shows the degree to which the VF was altered with respect to the original cadaveric vertebral bodies and the normal AVBs. The over-threshold and under-threshold specimens were adjusted to represent a change of $\pm 40 \%$ in the VF of the normal analogue, and this deviated by $+39 \%$ from the cadaveric sample. Fig. 9 shows the responses obtained from the different specimens under the same loading conditions. As expected, the printed specimen with the highest VF was the stiffest, with the normal showing good agreement with the real vertebral body. However, the closest specimen in terms of VF to the real vertebral body was the over-threshold analogue, and as expected this was considerably less stiff than the cadaveric sample (Table 4). This outcome was anticipated because bone is generally stiffer than ABS [18].

Low-resolution manufactured replicas were based on scans with a voxel dimension of $0.123 \mathrm{~mm}$ whereas the normal-resolution replicas had a voxel dimension of $0.063 \mathrm{~mm}$. Fig. 10 compares the stiffness values of the two analogues to the cadaveric sample. The dramatic increase in 
stiffness shown for the low-resolution analogue highlights the need for high-resolution CT scanning in order to recreate biofidelic replicas. This sharp increase in stiffness reflects the "bleaching" that occurs within the voxels. With higher scanning capability, darker areas become more evident against a lighter background, but as the voxel size increases so does the merging of the higher and lower GS values, resulting in a less defined geometry. When this is the reproduced by the 3D printer, which has only a single material capability, this bleached area will either be solid ABS or a void. The choice depends on the thresholding values, and whether the GS value falls within the selected range. This explains the increase in VF and consequently the stiffness.

\subsection{Tests of disc and facet joint compositions and facet loading}

As previously reported, the IVD plays a significant role in the initial part of the loading of the motion segment and a stiffer material was needed for a more realistic analogue response [7]. This was achieved by testing two different constitutions of two-part liquid rubber (PT Flex 85 and PT Flex 70), which provided Shore A hardness values of 85 and 70, respectively. Fig. 11 shows the responses of these materials compared to their cadaveric counterparts. In addition, AMS\#F denotes a fixed facet joint (in which the cartilage was simulated with the same grade of liquid rubber used for the IVD) and AMS\#NF denotes a non-fixed facet. When comparing the responses, we found that the facets play a key role in achieving more accurate and realistic representations of real vertebral bodies. Furthermore, PT Flex 85 provided a much better representation of the cadaveric response. This is shown clearly in Fig. 12, where the forcedisplacement curve of the biological sample lies between the curves representing the two types of rubber. All subsequent tests were therefore conducted using PT Flex 85.

Fig. 13 compares the stiffness values obtained using PT Flex 85 with facet fixation (FF) and the absence of facets (NFF). Again, the facet fixation experiment achieved more realistic stiffness values than the NFF analogues. DIC analysis also highlighted the significance of facet fixation in the accurate replication of responses. The analysis of initial loading and displacement with AMS4 revealed that the displacement observed for the whole motion segment (i.e. the vertebral body including the facet joint and IVD) was lower for the FF segment than the NFF segment. In 
addition, peak displacement occurred after approximately 6000 images, probably representing the stage at which the NFF specimen was compressed beyond the width of the joint and contact was made between the articular processes. The FF sample showed an immediate displacement and the overall displacement was greater than that observed in the NFF specimen, as shown in Fig. 14.

Finally, Fig. 15 shows the in-plane displacements observed for the AVB with facet fixation. Although the loading was uniaxial along the $y$ axis (compressive), an almost immediate in-plane displacement was detected on the lower AVB of the motion segment. In contrast, the facet displayed in-plane displacements much later, coinciding with the higher displacements along the $y$ axis. This is typical of the flexion movement of the spine, indicating that facets respond to loading in an interlinked manner. Although the facet showed increasing displacement, the AVB did not follow the same trend.

\section{Conclusions}

We have demonstrated that it is possible to fine-tune spinal motion segment analogues to obtain better, more biofidelic responses. Our data highlight the suitability of DIC data acquisition techniques, while confirming that any errors are strongly dependent on the settings, with the best results obtained using the point distance to facet size ratio recommended by GOM. In addition, the error trends in the dead weight experiments showed no conclusive relationship with the variation of the loading conditions.

The accuracy of printed analogues depends on the scanning resolution, given that a lowresolution scan overestimated the VF requirement. This is also true for thresholding techniques, which showed a similar effect to low scanning resolutions. The experiments described herein confirmed that the facet joint plays an integral part in the generation of results that are better and more closely related to real motion segments, and that the simulant used for the IVD should be selected carefully because it has a significant effect on the loading response of the whole segment. Although this research focused on quasi-static loading, it paves the way the development of rapid, inexpensive, accurate and replicable strategies for the preparation of patient-specific analogues. 


\section{Acknowledgments}

We acknowledge the work, skill and expertise of Karl Norris and the mechanical workshop of Cranfield University, Shrivenham, and Jolyon Cleaves of Vision Research for providing the high-speed cameras. Ethical approval was granted by the Cranfield University Research and Ethics committee (CURES). This paper is dedicated to our friend and colleague Dr Mike Gibson, whose untimely death is a great loss to us all.

\section{Data accessibility}

Data for this manuscript is available through the Cranfield University CORD data depository and preservation system (https://cranfield.figshare.com).

\section{Authors' contributions}

Conceptualisation: CF, PZ, IH, GT; Data curation: CF, EA, IH; Formal analysis: CF, EA, IH, MG; Funding acquisition: CF, PZ; Investigation: CF, EA; Methodology: CF, GT; Project administration: PZ; Resources: CF, IH; Software: CF, MG; Supervision: PZ, IH, GT; Validation: CF, GT; Visualisation: CF, GT; Writing (original draft): CF; Writing (review and editing): CF, EA, PZ.

\section{References}

1. Friss E A, Pence D C, Graber C D, Montoya J A. Mechanical analogue model of the human lumbar spine: development and evaluation. In: Melkerson M N, Griffith S L, Kirkpatric J S (eds) Spinal Implants: Are We Evaluating Them Appropriately? ASTM International, West Conshohocken, PA, USA, 2003, 236.

2. Wilke H J, Krischack S, Claes L E. Formalin fixation strongly influences biomechanical properties of the spine. Journal of Biomechanics, 1996, 29, 1629-31. 
3. Holsgrove T P, Gill H S, Miles A W, Gheduzzi S. The dynamic, six-axis stiffness matrix testing of porcine spinal specimens. The Spine Journal, 2015, 15, 176-84.

4. Costi J, Hearn T, Fazzalari N. The effect of hydration on the stiffness of intervertebral discs in an ovine model. Clinical Biomechanics, 2002, 17, 446-55.

5. Smeathers J E, Joanes D N. Dynamic compressive properties of human lumbar intervertebral joints: a comparison between fresh and thawed specimens. Journal of Biomechanics, 1988, 21, 425-33.

6. Sawbones. Biomechanical Spine Product Research, [2018-02-05], http://www.sawbones.com/UserFiles/Documents/Product/BioSpine_info.pdf.

7. Wang T, Ball J R, Pelletier M H, Walsh W R. Initial experience with synthetic spinal motion segments: biomechanical assessment of high cycle and implant performance. ORS Annual Meeting, New Orleans, LA, USA, 2014.

8. Domann J P. Development and Validation of an Analogue Lumbar Spine Model and its Integral Components. University of Kansas, Lawrence, KA, USA, 2011.

9. ASTM. Standard Test Methods for Spinal Implant Constructs in a Vertebrectomy Model. ASTM International, West Conshohocken, PA, USA, 2015.

10. Camisa W, Leasure J, Buckley J. Biomechanical validation of a synthetic lumbar spine. The Spine Journal, 2014, 14, 129-30.

11. Campbell J, Imsdahl S, Ching R. Evaluation of a synthetic L2-L5 spine model for biomechanical testing. Orthopaedic Research Society, New Orleans, 2010.

12. Franceskides C. Subject specific functional model of hard and soft tissues: Skull and Spine. $\mathrm{PhD}$ Thesis, Cranfield University, 2018.

13. Franceskides C, Arnold E, Horsfall I, Tozzi G, Gibson M.C, Zioupos P. Spinal Motion Segments - I: Concept for a Subject-specific Analogue Model. J Bionic Eng, 2020, (In Press)

14. Inglis S. 3D Printing in the NHS and healthcare sciences. IPEM Scope, 2016, 25, 10-13.

15. Cantrell J, Rohde S, Damiani D, Gurnani R, Di Sandro L, Anton J, Young A, Jerez A, Steinbach D, Kroese C, Ifju P. Experimental characterization of the mechanical properties of 3D-printed ABS and polycarbonate parts. Conference Proceedings of the Society for Experimental Mechanics Series, 2017, 3, 89-105. 
16. Zou R, Xia Y, Liu S, Hu P, Hou W, Hu Q, Shan C. Isotropic and anisotropic elasticity and yielding of 3D printed material. Composites Part B: Engineering, 2016, 99, 506-13.

17. Jaumard N V, Welch W C, Winkelstein B A. Spinal facet joint biomechanics and mechanotransduction in normal, injury and degenerative conditions. Journal of Biomechanical Engineering, 2011, 133, 071010.

18. Pal G P, Routal R V. Transmission of weight through the lower thoracic and lumbar regions of the vertebral column in man. Journal of Anatomy, 1987, 152, 93-105.

19. Shore A F, Shore C P. Apparatus for measuring the hardness of materials. US Patent US1770045 (A) - 1930-07-08, 1930.

20. Lott B D, Reece F N, Drott J H. Effect of preconditioning on bone breaking strength. Poultry Science, 1980, 59, 724-25.

21. Roberts S J, Smith C I, Millard A, Collins M J. The taphonomy of cooked bone: characterising boiling and its physico-chemical effects. Archaeometry, 2002, 44, 485-94.

22. Newell N, Little J P, Christou A, Adams M A, Adam C J, Masouros S D. Biomechanics of the human intervertebral disc: A review of testing techniques and results. Journal of the Mechanical Behavior of Biomedical Materials, 2017, 69, 420-34.

23. Palanca M, Tozzi G, Cristofolini L. The use of digital image correlation in the biomechanical area: a review. International Biomechanics, 2016, 3, 1-21.

24. Palanca M, Marco M, Ruspi M L, Cristofolini L. Full-field strain distribution in multivertebra spine segments: An in vitro application of digital image correlation. Medical Engineering \& Physics, 2018, 52, 76-83.

25. Vassolera JM and Fancelloa EA. Error analysis of the digital image correlation method. Asoc Argentina de Mecán Comp, 2012, 29, 6149-61.

26. Pan B, Lu Z and Xie H. Mean intensity gradient: An effective global parameter for quality assessment of the speckle patterns used in digital image correlation. Optics Lasers Eng 2010, 48, 469-77.

27. Lecompte D, Smits A, Bossuyt S, Sol H, van Tomme J, van Hemelrijck D, Habraken A M. Quality assessment of speckle patterns for digital image correlation. Optics Lasers Eng 2006, 44, 1132-45.

28. Sutton MA, Orteu JJ and Schreier H. Image correlation for shape, motion and deformation measurements. New York: Springer, 2009. 
29. Sutradhar A, Park J, Carrau D, Miller M.J., Experimental validation of 3D printed patientspecific implants using digital image correlation and finite element analysis. Comp Biol. Med. 2014, 52, 8-17.

30. Amiot F, Bornert M, Doumalin P, Dupré J- C, Fazzini M, Orteu J- J, Poilâne C, Robert L, Rotinat R, Toussaint E, Wattrisse B, Wienin J S. Assessment of digital image correlation measurement accuracy in the ultimate error regime: main results of a collaborative benchmark. Strain, 2013, 49, 483-96.

31. Siebert T, Becker T, Spiltthof K. Error estimations in digital image correlation technique. Applied Mechanics and Materials, 2007, 7, 265-70.

32. Zioupos P, Smith C, Yuehuei A. Factors affecting mechanical properties of bone. In: Yuehuei A, Robert D A (eds) Mechanical Testing of Bone and the Bone-Implant Interface. CRC Press, Boca Raton, FL, USA, 1999, 65-85

33. Smit T H. The use of a quadruped as an in vivo model for the study of the spine biomechanical considerations. European Spine Journal, 2002, 11, 137-44.

34. Busscher I, van der Veen A J, van Dieën J H. In vitro biomechanical characteristics of the spine: a comparison between human and porcine spinal segments. Spine, 2010, 35, 3542.

35. Dath R, Ebinesan A D, Porter K M, Miles A W. Anatomical measurements of porcine lumbar vertebrae. Clinical Biomechanics, 2007, 22, 607-13. 
Table 1 Allocation of analogues from cadaveric materials.

\begin{tabular}{|l|l|l|l|l|}
\hline Motion Segment & (L1-L2) & (T12-L1) & (L2-L3) & (L4-L5) \\
\hline Real motion segment & RMS 4 & RMS 5 & RMS 6 & RMS 7 \\
\hline \multirow{4}{*}{$\begin{array}{l}\text { ABS-based artificial } \\
\text { motion segment }\end{array}$} & AVB8_O & AVB5_N & AVB6_N & AVB7_N \\
& AMS8_70 & AVB5_L & AVB6_L & AVB7_L \\
& AMS8_85 & AMS5_F & AMS6_F & AMS7_F \\
& & AMSF & AMS6_NF & AMS7_NF \\
\hline
\end{tabular}

RMS: real (biological) motion segment; AMS: analogue (man-made) motion segment; AVB: artificial vertebral body; RMS\#_N : normal resolution scans 63 micron voxel size; RMS\#_L : low resolution scans 123 micron voxel size; AMS\#_F: with facet joints intact; AMS\#_NF: without facet joints; AVB\#_O: thresholding overcompensation increased volume fraction; AVB\#_U: thresholding undercompensation decreased Volume Fraction. F

Table 2 LCM point distance ratio compared to the facet sizes tested.

\begin{tabular}{|c|c|c|c|c|}
\hline \multicolumn{5}{|c|}{ Point distance to facet size ratio } \\
\hline $608 / 456$ & $456 / 456$ & $384 / 456$ & $228 / 456$ & $152 / 456$ \\
\hline 6 & 6 & 6 & 6 & 6 \\
\hline 12 & 12 & 12 & 12 & 12 \\
\hline 19 & 19 & 19 & 19 & 19 \\
\hline Not Comp & 24 & 24 & 24 & 24 \\
\hline Not Comp & 30 & 30 & 30 & 30 \\
\hline
\end{tabular}


Table 3 Volume fraction (VF) adjustments based on real vertebral bodies (RVB) and normal artificial vertebral bodies (AVB).

\begin{tabular}{|l|l|l|}
\hline \multicolumn{2}{|l|}{$\begin{array}{l}\text { Direction of } \\
\text { adjustment }\end{array}$} & VF adjustment \\
\hline \multirow{2}{*}{ RVB to } & Normal & 1.39 \\
& Over & 0.84 \\
& Under & 1.98 \\
\hline \multirow{2}{*}{ Normal to } & Over & 1.4 \\
& Under & 0.6 \\
\hline
\end{tabular}

Table 4 Stiffness values for real vertebral bodies (RVB) and artificial vertebral bodies (AVB).

\begin{tabular}{|l|l|}
\hline Specimen & Stiffness N/mm \\
\hline AVB Over & 17429 \\
AVB Norm & 10808 \\
AVB Under & 7775 \\
RVB & 9778 \\
\hline
\end{tabular}




\section{FIGURE CAPTIONS}

Fig. 1 PVC core setup. (a) PVC core with strain gauge attached and speckle pattern applied; (b) Detailed GOM configuration.

Fig. 2 Dead weight testing apparatus, with red block denoting weight, black rods denoting the traverse guides and the grey block denoting the sample.

Fig. 3 Artificial vertebral bodies as a motion segment and in different illustrations. (a) with the speckled pattern to allow use of DIC (optical tracking of the deformations); (b) microCT scan of the actual real biological motion segment thresholded to show the denser bone parts (vertebrae) and thus showing a gap where the intervertebral disk is situated between the 2 vertebrae; (c) microCT scan illustration of the artificially created analogue model (same as in (a)) with different colours used to show the 2 vertebrae and the disk in between them; (d) cross section in the microCT scan showing the inner structure of the analogue model produced by the 3D printing (printed and rescanned sample) with support material highlighted in green, and ABS shown in grey.

Fig. 4 (a) Error variance between the strain gauge data and each point distance to facet size ratio, with varying facet size. (b) Error variance between the strain gauge data and each facet size, with varying point distance to facet size ratio. AV: average value of data.

Fig. 5 Strain measurements obtained by DIC analysis compared to extensometer strain gauge data, with the difference (error) shown as a dashed line.

Fig. 6 Static error over a viewing aperture of $20 \times 20 \mathrm{~mm}$ on the $x$ and $y$ axes.

Fig. 7 The $y$-axis displacement error under uniaxial dead weight loading.

Fig. 8 Effect of support cleaning apparatus on artificial vertebral bodies under compressive loading.

Fig. 9 Load-displacement curves of normal, over-threshold and under-threshold artificial vertebral bodies compared to a real cadaveric sample. 
Fig. 10 Scanning resolution effects for three artificial vertebral bodies compared to a real cadaveric sample.

Fig. 11 Comparison of two-part silicone composition (PTFlex 70 LHS and PTFlex85 RHS) with and without facet fixation.

Fig. 12 AMS5 force-displacement curve with and without facet fixation, compared to a cadaveric motion segment.

Fig. 13 Effect of facet fixation on the loading response of four motion segments.

Fig. 14 DIC analysis of displacements for entire motion segments and their associated facets using fixed facet (FF) and non-fixed facet (NFF) samples.

Fig. 15 In-plane displacements for the bottom of an artificial vertebral body and its associated fixed facet joint. 


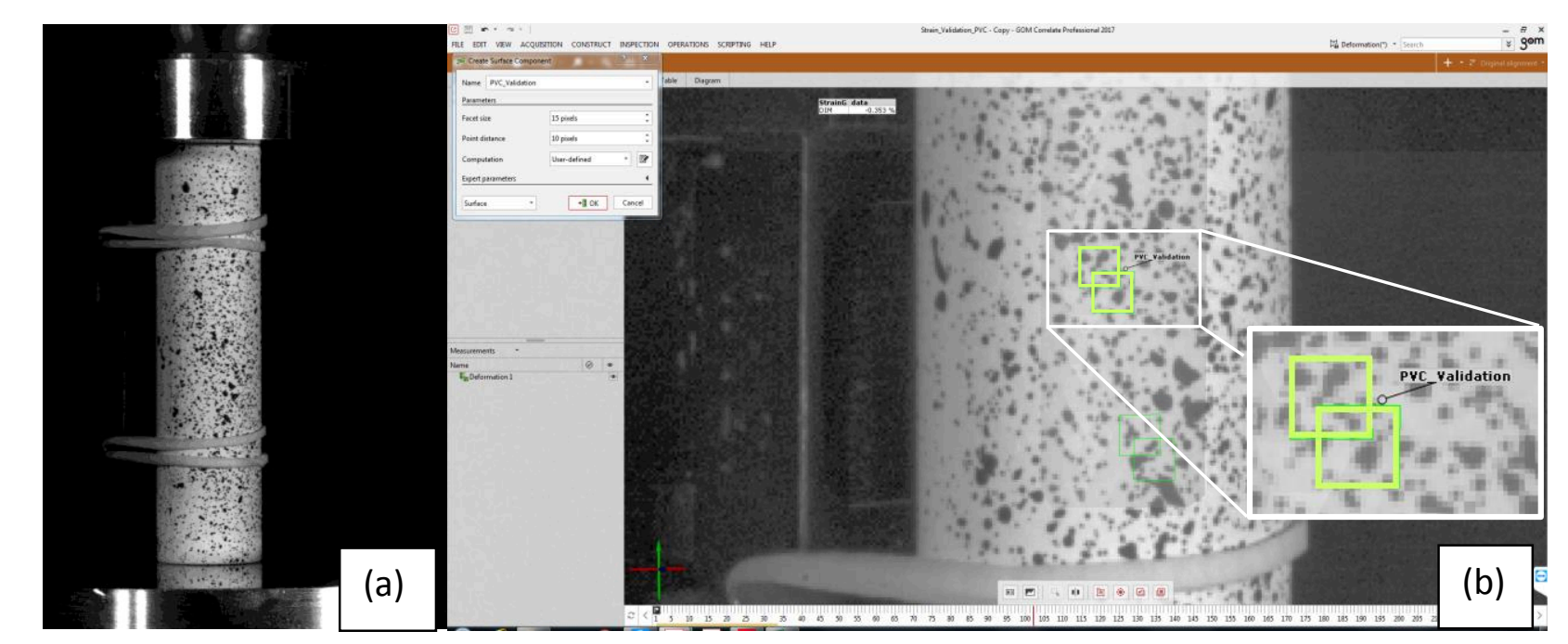

Fig -1

ig -1 


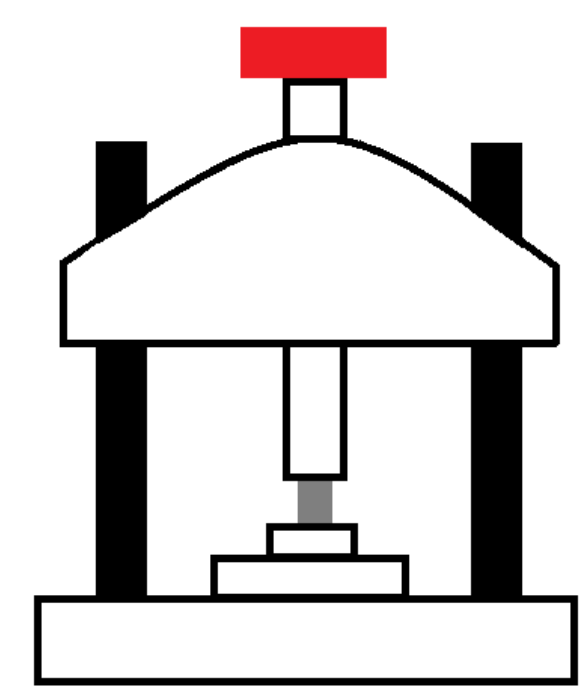

Fig -2

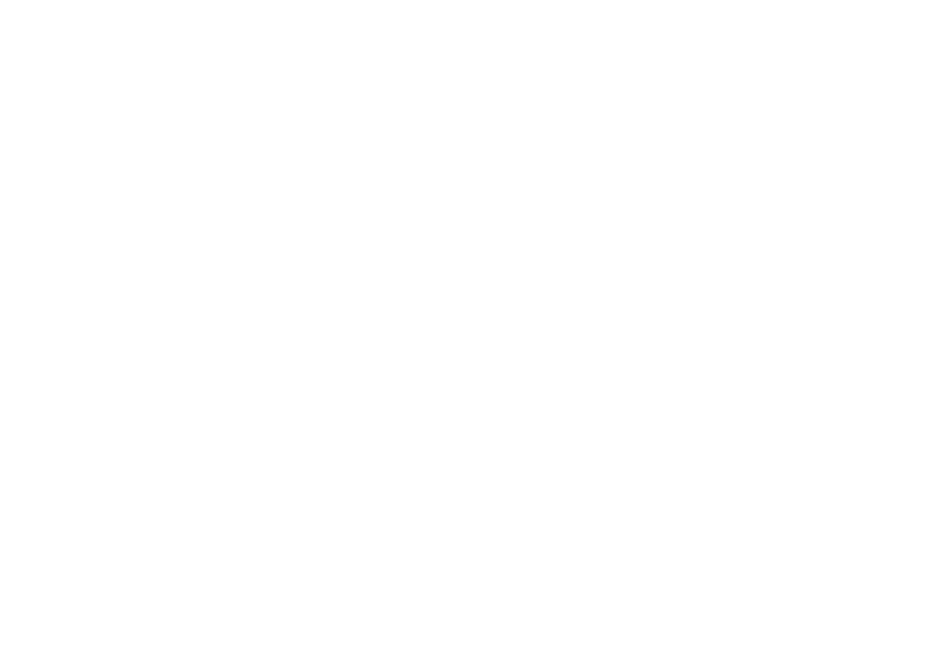



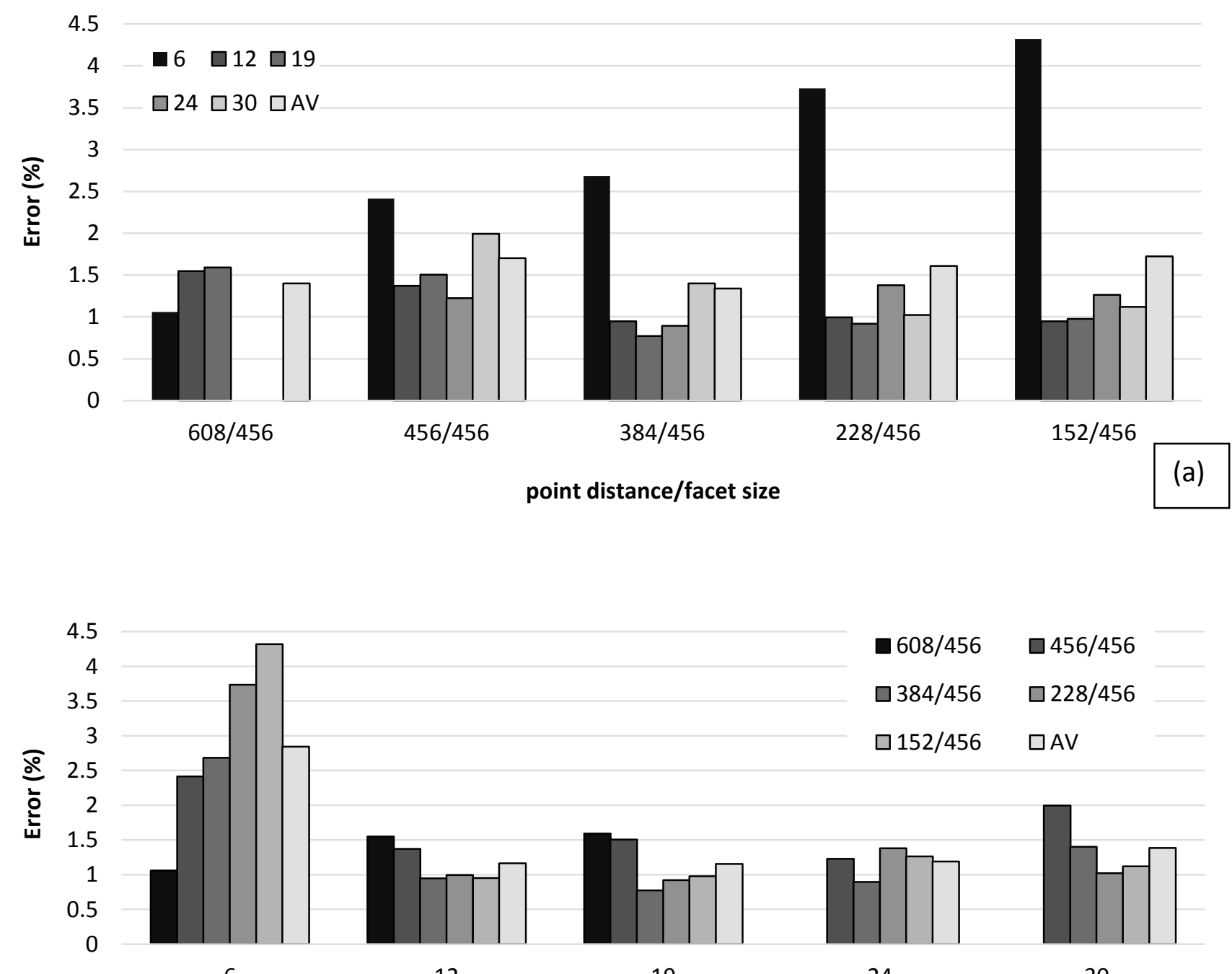

6

$\begin{array}{ll}\square 608 / 456 & \square 456 / 456 \\ \square 384 / 456 & \square 228 / 456 \\ \square 152 / 456 & \square A V\end{array}$

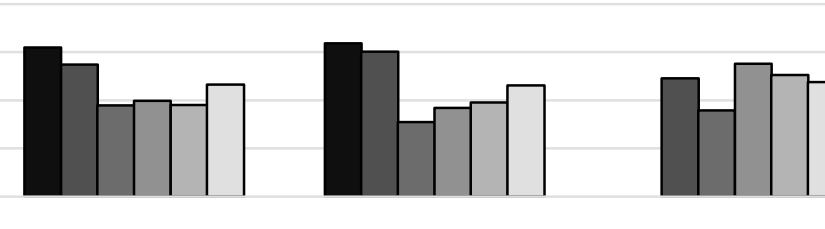

24

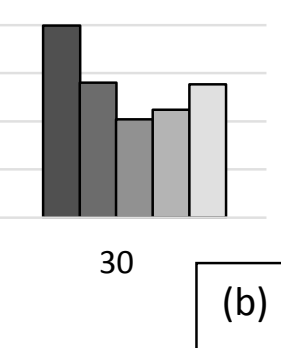

Fig -4 


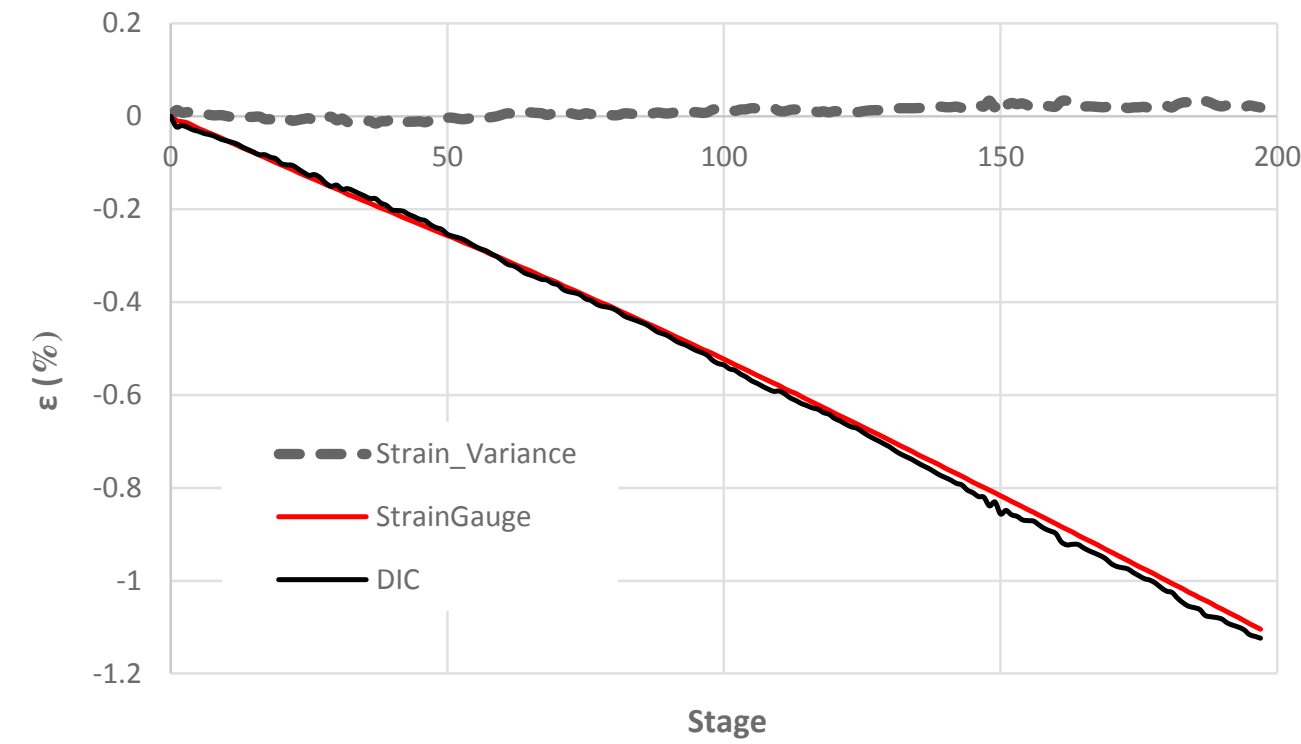

Fig -5

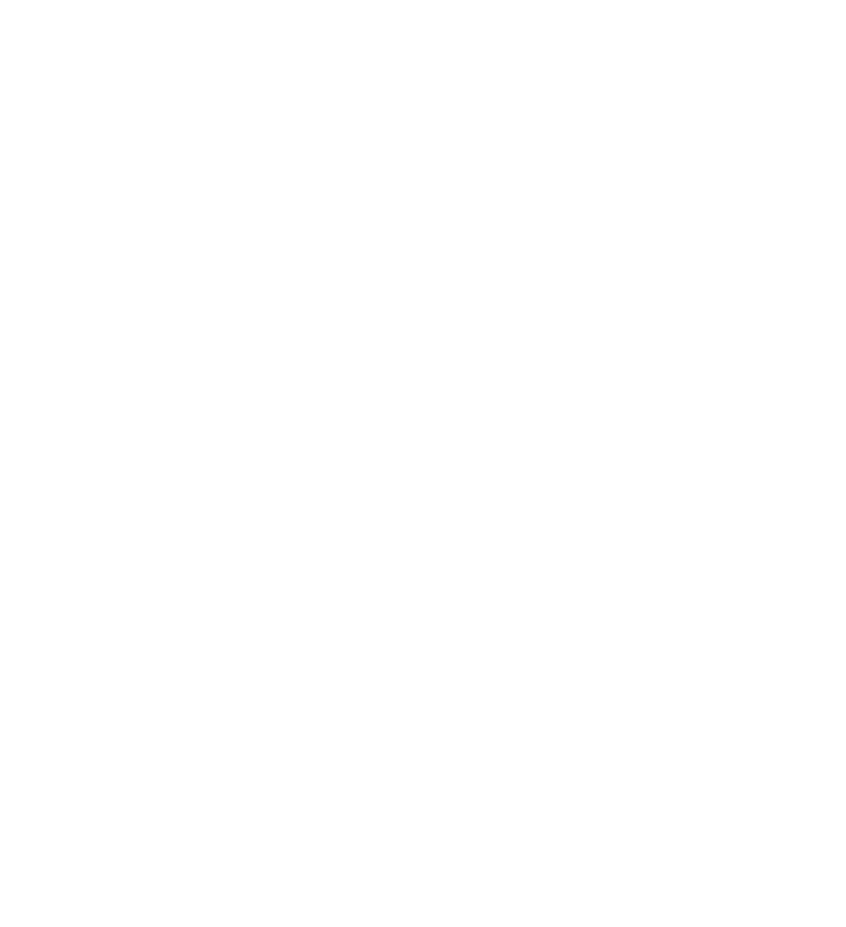

Stage 


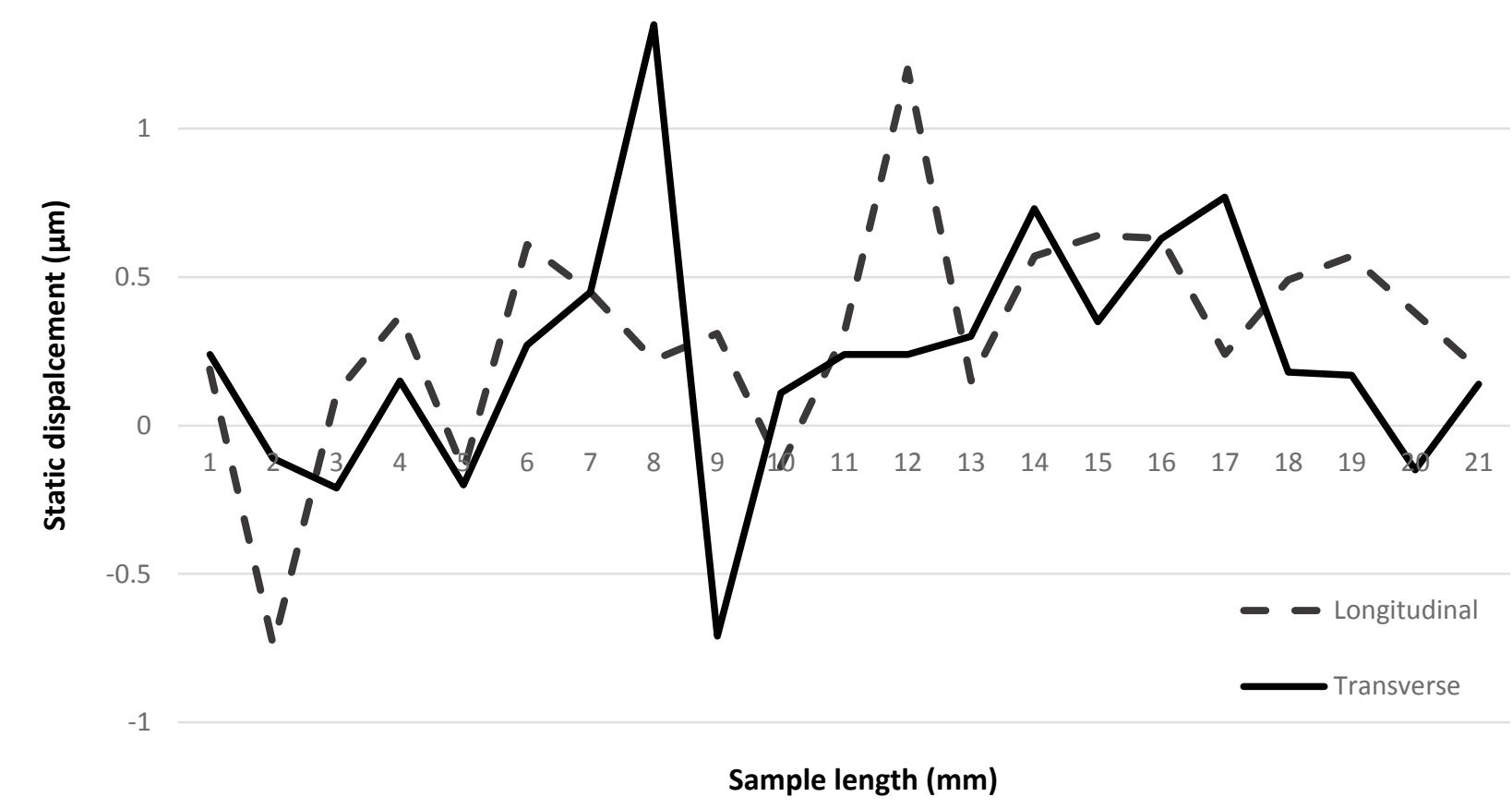

Fig -6 


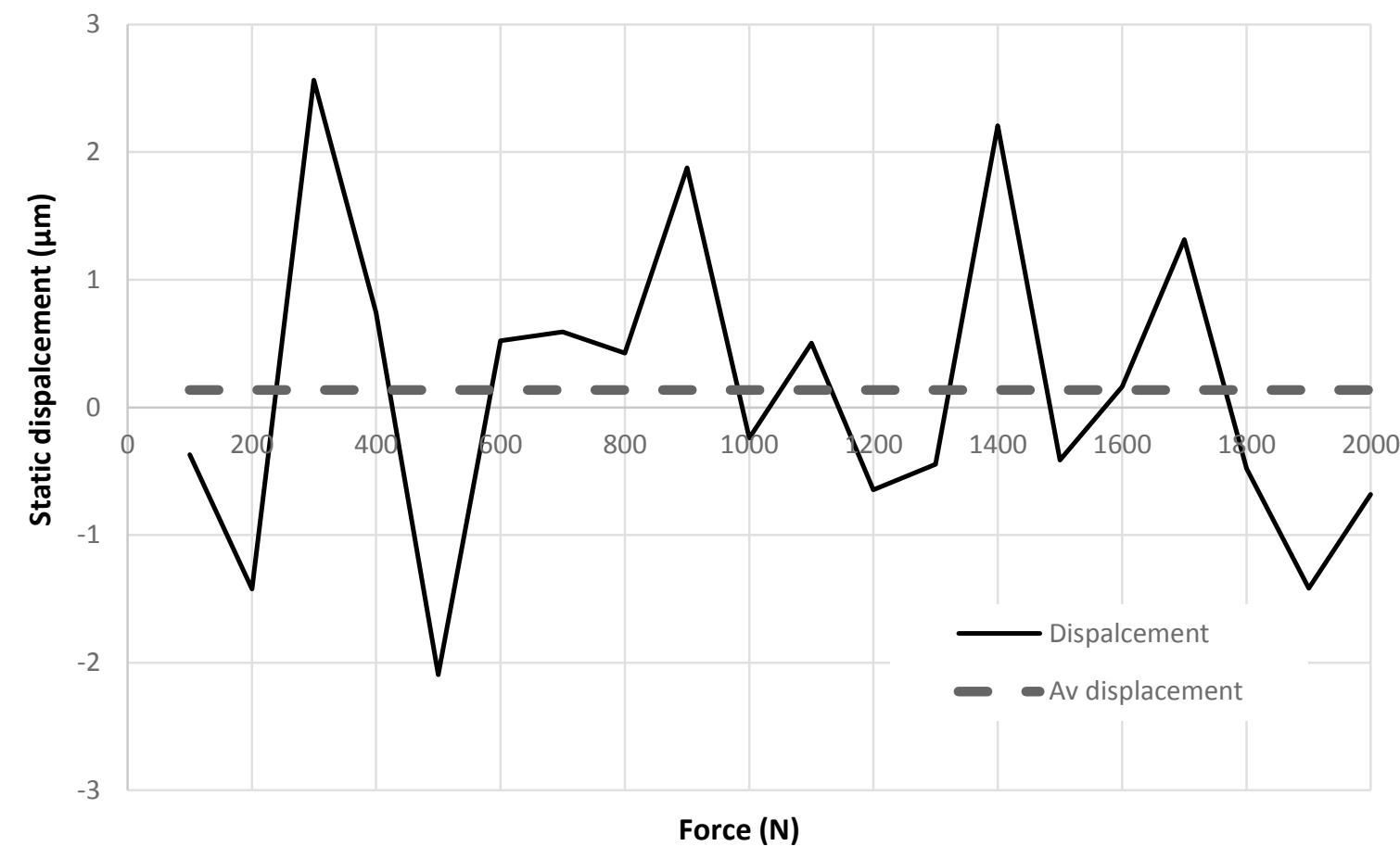

Fig -7

Force (N)

\section{Fig -7}




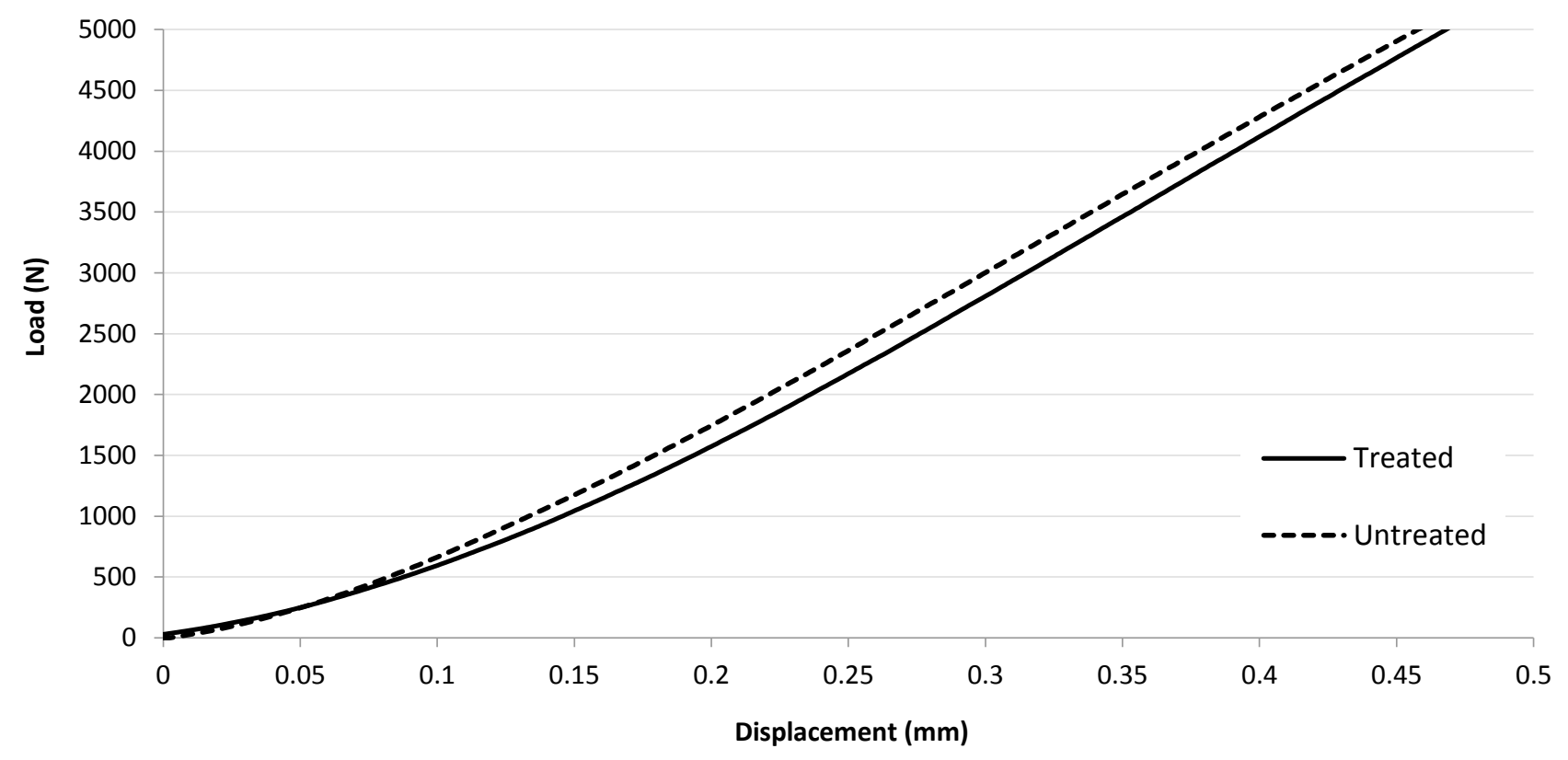

Fig -8

Displacement (mm)

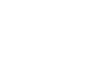


Figure 9

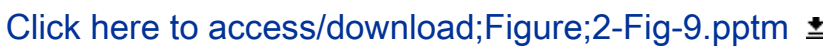

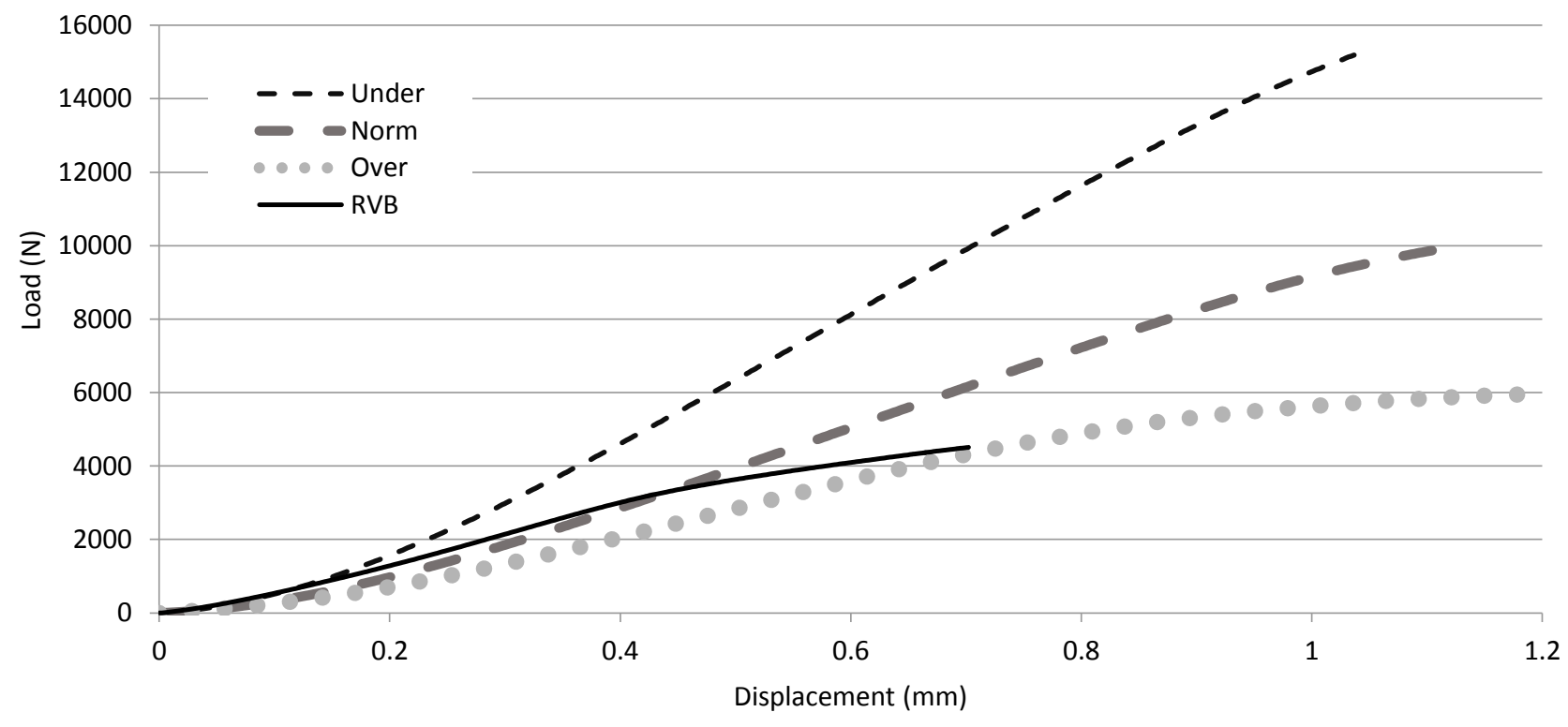

Fig -9 


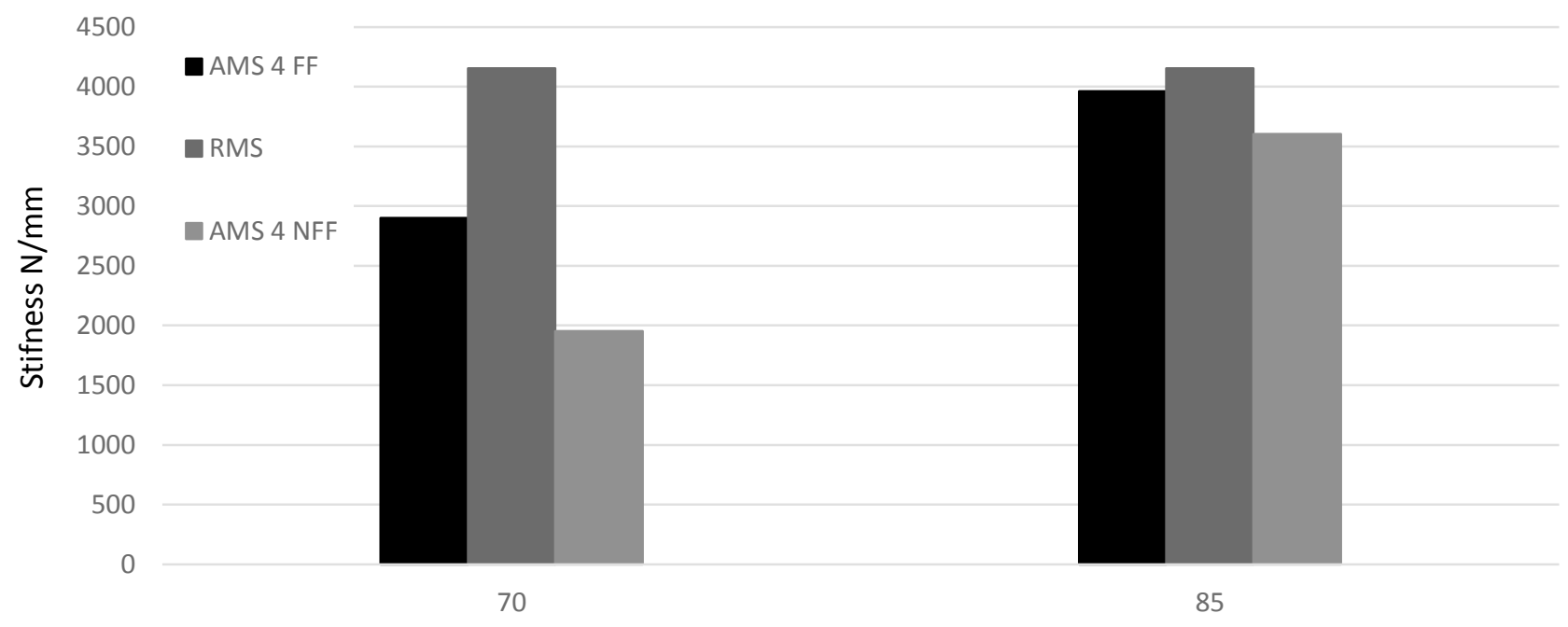

Fig -11 


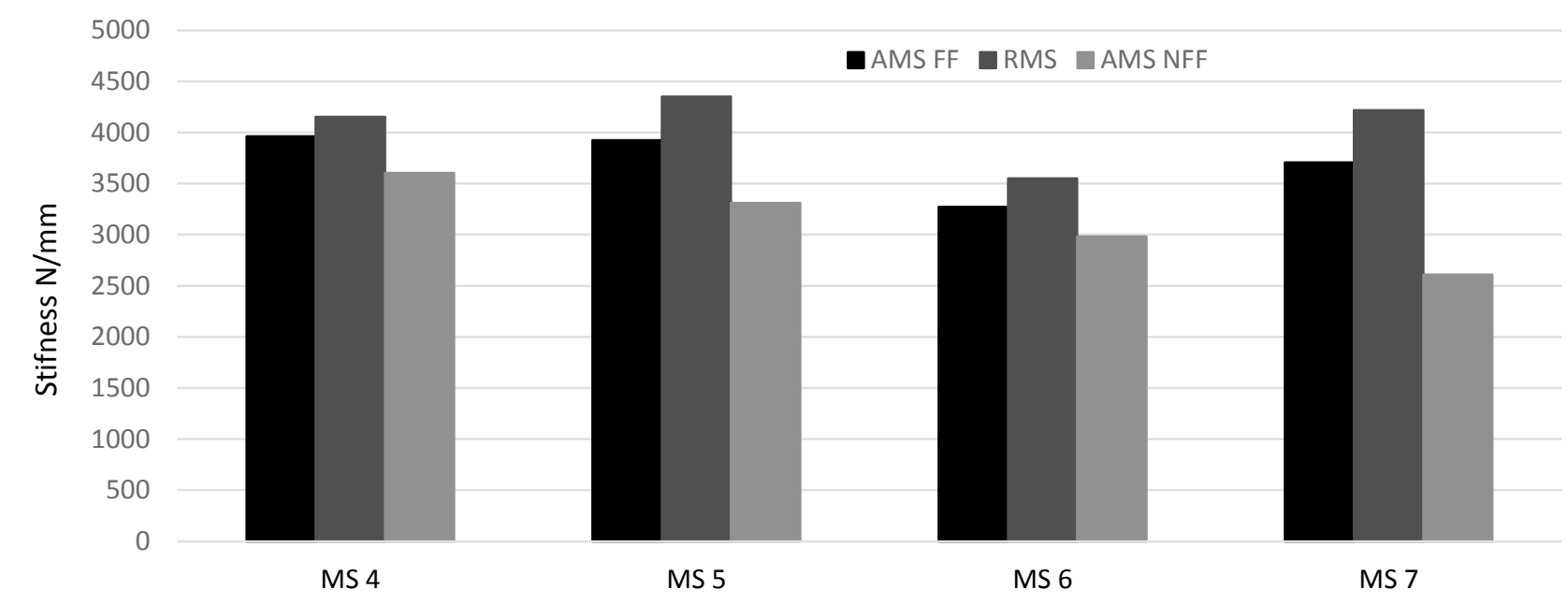

Fig -13 
$\begin{array}{llllllllllllll}0 & 1500 & 3000 & 4500 & 6000 & 7500 & 9000 & 10500 & 12000 & 13500 & 15000 & 16500 & 18000 & 19500\end{array}$

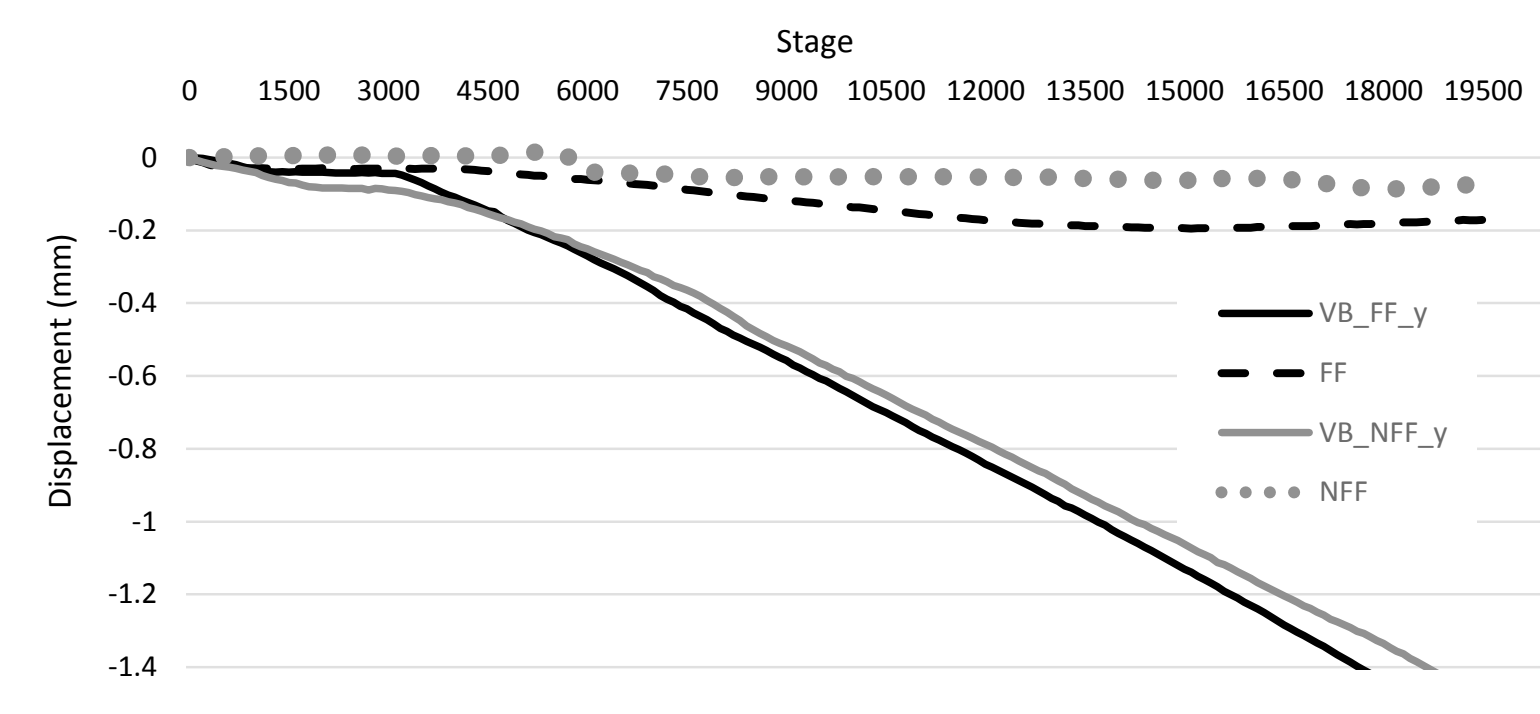

Fig -14

$-1.4$

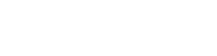

\section{4}




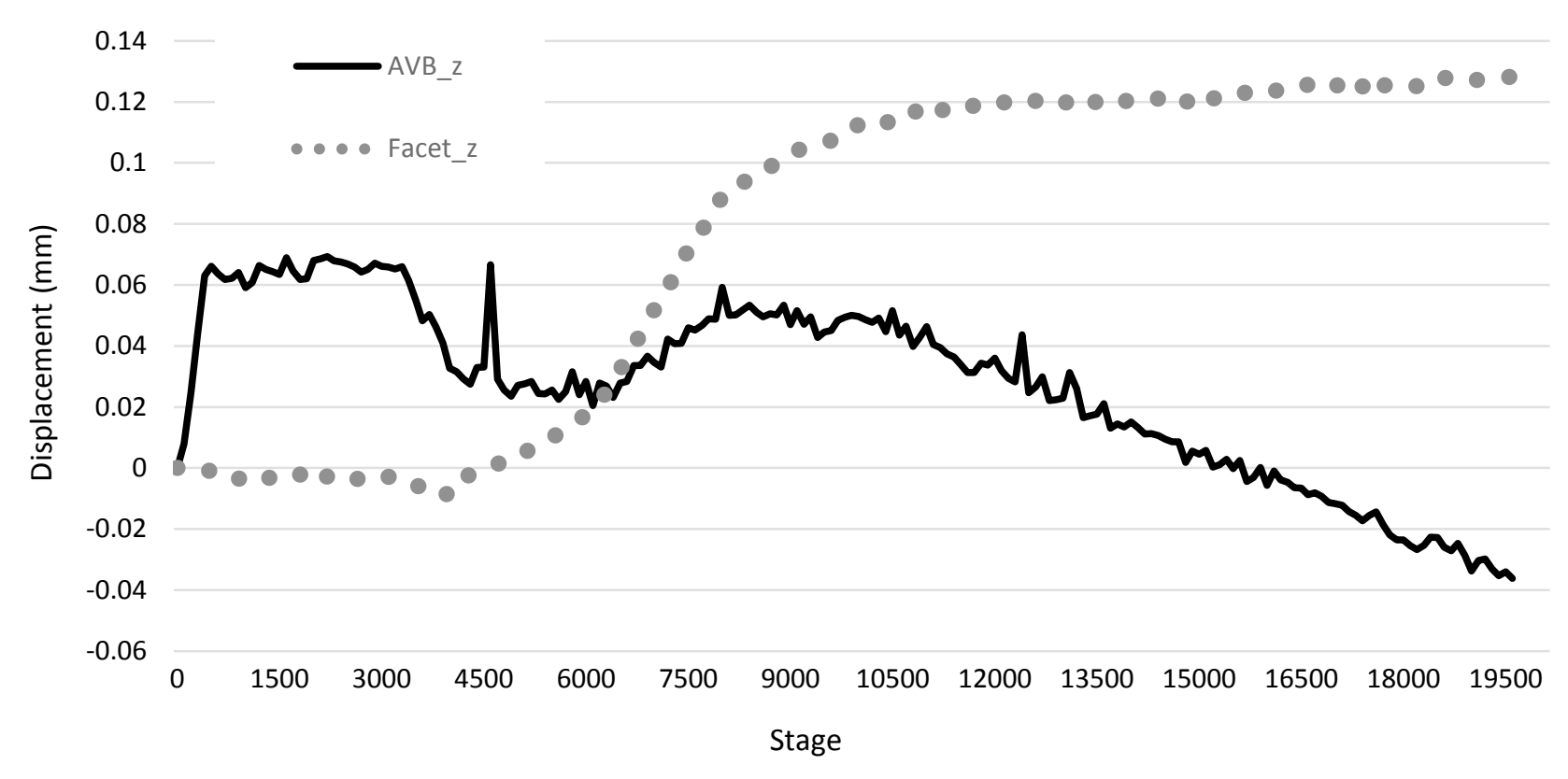

Fig -15 5 